\title{
PREGNANCY-ASSOCIATED BREAST CANCER: ANALYSIS OF CASES IN THE MASTOLOGY SERVICE OF HOSPITAL SANTA MARCELINA DE ITAQUERA, SÃO PAULO, FROM 2014 TO 2019
}

Grasiela Benini dos Santos Cardoso'

${ }^{1}$ Hospital Santa Marcelina - São Paulo (SP), Brazil.

Thirty-one year old, median gestational age of 28 weeks. Four of our patients were primiparous with less than 20 years of age, and two between the ages of 30 and 34, with median of secundiparous patients (50\%). By analyzing the obesity and BMI (body mass index) factors, we concluded that 3 patients were mildly obese, and one presented with morbid obesity at the time of diagnosis. Our BMI mean was 29. Considering histology and immunohistochemical, eight patients were diagnosed with ductal invasive carcinoma, without other specifications; one was diagnosed with fusiform cell carcinoma, and one with mucinous carcinoma. The histological subtypes found were luminal B (4 cases) and triple negative (6 cases). These results were compatible with a French retrospective study from 2017. The pregnant woman, or in the puerperium, with breast cancer may present the same symptoms as the other patients with the disease, but diagnosis can be delayed due to the physiological changes in breast tissue in the pregnancy-puerperal period. In our study, all patients were diagnosed at advanced clinical staging (IIIA, IIIB and IV). The treatment follows the same protocols as for non-pregnant patients, considering not only the type of tumor and disease staging, but also gestational age. The most used therapy for our group was neoadjuvant chemotherapy, followed by radical modified mastectomy. This was owed to the advanced stage of the disease. The sentinel lymph node biopsy was performed in two patients. One was diagnosed in the post-partum period, and the other was diagnosed while pregnant of 34 weeks. The latter received surgical treatment after the pregnancy. Chemotherapics are relatively safe when applied after the second trimester. During the patients' follow-up, one of them presented with progression of the disease to the brain (this patient was in stage IV, with lung metastasis); one presented with bone and hepatic metastasis; and the other one had plastron recurrence. Until the conclusion of this study, four patients died. Conclusions: Pregnancy-associated breast cancer is a condition that should be observed by health teams, since its early diagnosis enables an approach that minimizes damages for the maternal-fetal binomial. Besides, detecting this disease in its early stages is the main factor that impacts the disease-free survival. 